\title{
On expansions of weakly o-minimal non-valuational structures by convex predicates
}

by

\author{
Roman Wencel (Wrocław)
}

\begin{abstract}
We prove that if $\mathcal{M}=(M, \leq,+, \ldots)$ is a weakly o-minimal non-valuational structure expanding an ordered group $(M, \leq,+)$, then its expansion by a family of "nonvaluational" unary predicates remains non-valuational. The paper is based on the author's earlier work on strong cell decomposition for weakly o-minimal non-valuational expansions of ordered groups.
\end{abstract}

0. Introduction. Several examples of weakly o-minimal structures are obtained as expansions of o-minimal structures by predicates interpreted as certain convex sets [MMS]. Among these we have an expansion of a real closed field by a valuation ring and an expansion of the ordered field of real algebraic numbers by a predicate interpreted as $(-\alpha, \alpha)$, where $\alpha$ is a transcendental number. Structures of the first sort were investigated by L. van den Dries and A. H. Lewenberg (see [DL] and [D2]) who showed for instance that if $\mathcal{R}$ is an o-minimal expansion of a real closed field and $V$ is a proper non-empty convex subring closed under 0-definable continuous functions, then the expansion $(\mathcal{R}, V)$ is weakly o-minimal. The phenomenon occuring in all the mentioned examples was addressed in general by Y. Baisalov and B. Poizat (see $[\mathrm{BP}]$ ) who proved that an expansion of any o-minimal structure by a family of convex predicates has weakly o-minimal theory (so in particular it is a weakly o-minimal structure). The result of [BP] was generalized by B. Baizhanov (see [Bz]) who proved that an expansion of a model of a weakly o-minimal theory by a family of convex predicates has weakly o-minimal theory. It is worth mentioning that Baizhanov's theorem also has

2000 Mathematics Subject Classification: Primary 03C64.

Key words and phrases: weak o-minimality, expansions by predicates.

This research was supported by a Marie Curie Intra-European Fellowship within the 6th European Community Framework Programme (contract number: MEIF-CT-2003501326) and by a Marie Curie European Reintegration Grant (contract number MERGCT-2005-031095). 
an easy proof when one uses the fact that weakly o-minimal theories do not have the independence property and the result of S. Shelah (see [Sh783]) concerning quantifier elimination for the theory of an expansion of a sufficiently saturated model of a theory without the independence property by all externally definable sets. The question of G. Cherlin whether every expansion of a weakly o-minimal structure (not necessarily with weakly o-minimal theory) by a family of convex predicates is also weakly o-minimal still remains an open problem.

This paper is a sequel to the study of expansions of weakly o-minimal structures by convex predicates. In [We07] I introduced weakly o-minimal non-valuational expansions of ordered groups as a natural generalization of weakly o-minimal non-valuational expansions of real closed fields considered in [MMS]. A weakly o-minimal expansion of a real closed field is said to be non-valuational iff it does not define a non-trivial valuation. Similarly, a weakly o-minimal expansion $\mathcal{M}$ of an ordered group $(M, \leq,+)$ is called non-valuational (or of non-valuational type) iff there is no proper and nontrivial subgroup of $(M,+)$ definable in $\mathcal{M}$. Being of non-valuational type is equivalent to several conditions which are discussed in [We07]. One of them says that the distance between the two parts of a definable cut is zero (the precise definition appears in $\S 1)$.

Assume that $\mathcal{M}=(M, \leq,+, \ldots)$ is a weakly o-minimal non-valuational expansion of an ordered group. If $P \subseteq M$ is a finite union of convex sets, then $P$ in a natural way determines a finite family of cuts in $(M, \leq)$. If the parts of all these cuts are "close" to each other, then $P$ is said to be non-valuational. Moreover, the expansion $(\mathcal{M}, P)$ is interdefinable with some expansion of $\mathcal{M}$ by a family of convex predicates. By [We07], $\mathrm{Th}(\mathcal{M})$ is weakly o-minimal. Therefore by $[\mathrm{Bz}]$ also any expansion of $\mathcal{M}$ by a family of non-valuational predicates has weakly o-minimal theory. The main result of this paper is Theorem 2.11, which says that every expansion of $\mathcal{M}$ by a family of nonvaluational predicates is of non-valuational type. We also show that the theory of such an expansion is weakly o-minimal without using Baizhanov's theorem (cf. Corollary 2.10).

The paper is organized as follows. In $\S 1$ we fix our notation and terminology, and recall some particularly useful results, mainly from [MMS] and [We07]. In $\S 2$ we outline the proofs of the results mentioned above.

Last but not least, I would like to thank the referee whose comments allowed me to improve the quality of the paper.

1. Notation and preliminaries. Let $(M, \leq)$ be a dense linear ordering without endpoints. A set $I \subseteq M$ is called convex in $(M, \leq)$ iff for any $a, b \in I$ and $c \in M$ with $a \leq c \leq b$ we have $c \in I$. If additionally $I \neq \emptyset$ and 
$\inf I, \sup I \in M \cup\{-\infty,+\infty\}$, then $I$ is called an interval in $(M, \leq)$. A maximal convex subset of a non-empty subset of $M$ is called a convex component of it. A pair $\langle C, D\rangle$ of non-empty subsets of $M$ is called a cut in $(M, \leq)$ iff $C<D, C \cup D=M$ and $D$ has no lowest element. A first order structure $\mathcal{M}=(M, \leq, \ldots)$ expanding $(M, \leq)$ is said to be weakly o-minimal iff every subset of $M$, definable in $\mathcal{M}$, is a finite union of convex sets. A complete first order theory is called weakly o-minimal iff all its models are weakly o-minimal. The following remark characterizes weakly o-minimal structures in terms of sets definable in them.

REMARK 1.1. Assume that $(M, \leq)$ is a dense linear ordering without endpoints and for $m \in \mathbb{N}_{+}, \mathcal{D}_{m}$ is a family of subsets of $M^{m}$ for which the following conditions are satisfied.

(a) If $I \subseteq M$ is an interval, then $I \in \mathcal{D}_{1}$.

(b) If $X \in \mathcal{D}_{1}$, then $X$ is a union of finitely many convex sets.

(c) $\left\{\langle x, y\rangle \in M^{2}: x<y\right\} \in \mathcal{D}_{2}$.

(d) $\mathcal{D}_{m}$ with the usual set-theoretic operations is a Boolean algebra.

(e) If $X \in \mathcal{D}_{m}$, then $X \times M, M \times X \in \mathcal{D}_{m+1}$.

(f) If $X \in \mathcal{D}_{m+1}$ and $\pi: M^{m+1} \rightarrow M^{m}$ is the projection dropping the last coordinate, then $\pi[X] \in \mathcal{D}_{m}$.

(g) If $1 \leq i<j \leq m$, then $\left\{\left(x_{1}, \ldots, x_{m}\right) \in M^{m}: x_{i}=x_{j}\right\} \in \mathcal{D}_{m}$.

Then there is a weakly o-minimal structure $\mathcal{M}$ expanding $(M, \leq)$ such that for every $X \subseteq M^{m}, X$ is definable in $\mathcal{M}$ iff $X \in \mathcal{D}_{m}$.

Note that if one replaces "convex sets" in (b) with "intervals", then (a)-(g) in Remark 1.1 imply that there is an o-minimal expansion $\mathcal{M}$ of $(M, \leq)$ such that for every $X \subseteq M^{m}, X$ is definable in $\mathcal{M}$ iff $X \in \mathcal{D}_{m}$ (cf. [D1, Chapter 1]).

Assume that $\mathcal{M}=(M, \leq, \ldots)$ is a weakly o-minimal structure. A cut $\langle C, D\rangle$ in $(M, \leq)$ is called definable in $\mathcal{M}$ iff the sets $C, D$ are definable in $\mathcal{M}$. The set of all such cuts will be denoted by $\bar{M}^{\mathcal{M}}$. The set $M$ can be regarded as a subset of $\bar{M}^{\mathcal{M}}$ by identifying an element $a \in M$ with the cut $\langle(-\infty, a],(a,+\infty)\rangle$. After such an identification, $\bar{M}^{\mathcal{M}}$ is naturally equipped with a dense linear ordering without endpoints extending that of $(M, \leq)$, and $(M, \leq)$ is dense in $\left(\bar{M}^{\mathcal{M}}, \leq\right)$. For a definable set $X \subseteq M^{m}$, a function $f: X \rightarrow \bar{M}^{\mathcal{M}}$ is said to be definable in $\mathcal{M}$ iff the set $\{\langle\bar{x}, y\rangle \in X \times M$ : $f(\bar{x})>y\}$ is definable in $\mathcal{M}$.

Now assume that $\mathcal{M}=(M, \leq,+, \ldots)$ is a weakly o-minimal structure expanding an ordered group $(M, \leq,+)$. A cut $\langle C, D\rangle$ is called non-valuational $\operatorname{iff} \inf \{y-x: x \in C, y \in D\}=0$. We will say that $\mathcal{M}$ is non-valuational (or of non-valuational type) iff all cuts definable in $\mathcal{M}$ are non-valuational. If $\mathcal{M}$ is non-valuational, then $\bar{M}^{\mathcal{M}}$ can be naturally equipped with an ordered 
group structure extending that of $(M, \leq,+)$. The ordered groups $(M, \leq,+)$ and $\left(\bar{M}^{\mathcal{M}}, \leq,+\right)$ are divisible, abelian and torsion free. For details we refer the reader to $\S 1$ of [We07].

Assume that $\mathcal{M}=(M, \leq, \ldots)$ is a weakly o-minimal structure. Below, for every $m \in \mathbb{N}_{+}$we inductively define strong cells in $M^{m}$ and their completions in $\left(\bar{M}^{\mathcal{M}}\right)^{m}$. The completion in $\left(\bar{M}^{\mathcal{M}}\right)^{m}$ of a strong cell $C \subseteq M^{m}$ will be denoted by $\bar{C}$.

(1) A one-element subset of $M$ is a strong $\langle 0\rangle$-cell in $M$ and is equal to its completion.

(2) A non-empty convex open definable subset of $M$ is a strong $\langle 1\rangle$-cell in $M$. If $C \subseteq M$ is a strong $\langle 1\rangle$-cell in $M$, then $\bar{C}:=\left\{x \in \bar{M}^{\mathcal{M}}\right.$ : $(\exists a, b \in C)(a<x<b)\}$.

Assume that $m \in \mathbb{N}_{+}, i_{1}, \ldots, i_{m} \in\{0,1\}$ and suppose that we have already defined strong $\left\langle i_{1}, \ldots, i_{m}\right\rangle$-cells in $M^{m}$ and their completions in $\left(\bar{M}^{\mathcal{M}}\right)^{m}$.

(3) If $C \subseteq M^{m}$ is a strong $\left\langle i_{1}, \ldots, i_{m}\right\rangle$-cell in $M^{m}$ and $f: C \rightarrow M$ is a continuous definable function which has a continuous extension $\bar{f}: \bar{C} \rightarrow \bar{M}^{\mathcal{M}}$, then $\Gamma(f)$, the graph of $f$, is a strong $\left\langle i_{1}, \ldots, i_{m}, 0\right\rangle$-cell in $M^{m+1}$. The completion of $\Gamma(f)$ in $\left(\bar{M}^{\mathcal{M}}\right)^{m+1}$ is defined as $\Gamma(\bar{f})$.

(4) If $C \subseteq M^{m}$ is a strong $\left\langle i_{1}, \ldots, i_{m}\right\rangle$-cell in $M^{m}$ and $f, g: C \rightarrow \bar{M}^{\mathcal{M}} \cup$ $\{-\infty,+\infty\}$ are continuous definable functions which have continuous extensions $\bar{f}, \bar{g}: \bar{C} \rightarrow \bar{M}^{\mathcal{M}}$ such that $\bar{f}(\bar{x})<\bar{g}(\bar{x})$ for $\bar{x} \in \bar{C}$, then the set

$$
(f, g)_{C}:=\{\langle\bar{a}, b\rangle \in C \times M: f(\bar{a})<b<g(\bar{a})\}
$$

is called a strong $\left\langle i_{1}, \ldots, i_{m}, 1\right\rangle$-cell in $M^{m}$. The completion of $(f, g)_{C}$ in $\left(\bar{M}^{\mathcal{M}}\right)^{m+1}$ is defined as

$$
\overline{(f, g)_{C}}:=(\bar{f}, \bar{g})_{\bar{C}}:=\left\{\langle\bar{a}, b\rangle \in \bar{C} \times \bar{M}^{\mathcal{M}}: \bar{f}(\bar{a})<b<\bar{g}(\bar{a})\right\} .
$$

(5) We say that $C \subseteq M^{m}$ is a strong cell in $M^{m}$ iff there are $i_{1}, \ldots, i_{m} \in$ $\{0,1\}$ such that $C$ is a strong $\left\langle i_{1}, \ldots, i_{m}\right\rangle$-cell in $M^{m}$.

If $C \subseteq M^{m}$ is a strong cell, then a definable function $f: C \rightarrow \bar{M}^{\mathcal{M}}$ is called strongly continuous iff $f$ has a continuous extension $\bar{f}: \bar{C} \rightarrow \bar{M}^{\mathcal{M}}$. In a standard way we define the notion of decomposition of a definable set into strong cells partitioning a given definable set (for details we refer the reader to $\S 2$ of [We07]). We will say that $\mathcal{M}$ has the strong cell decomposition property iff for any $m, k \in \mathbb{N}_{+}$and any definable sets $X_{1}, \ldots, X_{k} \subseteq M^{m}$, there is a decomposition of $M^{m}$ into strong cells partitioning each of the sets $X_{1}, \ldots, X_{k}$.

FACT 1.2 ([MMS, Ar]). Let $\mathcal{M}=(M, \leq, \ldots)$ be a weakly o-minimal structure and $A \subseteq M$. If $U \subseteq M$ is an infinite $A$-definable set and $f$ : 
$U \rightarrow \bar{M}^{\mathcal{M}}$ is an A-definable function, then there is a partition of $U$ into $A$ definable sets $X, I_{0}, \ldots, I_{m}$ such that $X$ is finite, $I_{0}, \ldots, I_{m}$ are non-empty convex open sets, and for every $i \leq m, f\left\lceil I_{i}\right.$ is locally constant or locally strictly increasing or locally strictly decreasing.

The following fact easily follows from the definition of strong cells.

FACT 1.3. Assume that $\mathcal{M}=(M, \leq, \ldots)$ is a weakly o-minimal structure with the strong cell decomposition property and $A \subseteq M$. If $U \subseteq M$ is a nonempty A-definable set and $f: U \rightarrow \bar{M}^{\mathcal{M}}$ is an A-definable function, then there is a partition of $U$ into $A$-definable sets $X, I_{0}, \ldots, I_{m}$ such that $X$ is finite, $I_{0}, \ldots, I_{m}$ are non-empty convex open sets, and for every $i \leq m, f\left\lceil I_{i}\right.$ is strongly continuous and strictly monotone or constant.

Proof. By assumption, there is a decomposition $\mathcal{C}$ of $M^{2}$ into $A$-definable strong cells partitioning the set $\{\langle x, y\rangle \in U \times M: y<f(x)\}$. This yields a decomposition of $U$ into finitely many $A$-definable convex open sets $J_{0}, \ldots, J_{k}$ and a finite set $X$ such that

$$
\left\{J_{0}, \ldots, J_{k}\right\} \cup\{\{a\}: a \in X\}=\{\pi[C]: C \in \mathcal{C}\},
$$

where $\pi: M^{2} \rightarrow M$ is the projection dropping the second coordinate. By our definition of strong cells, $f \nmid J_{i}$ is strongly continuous for $i \leq m$. In such a situation, weak o-minimality of $\mathcal{M}$ implies that each of the $J_{i}$ 's could be decomposed into finitely many $A$-definable convex open sets and some finite set so that on each of the open sets, $f$ is strictly monotone or constant. This finishes the proof.

TheOrem 1.4 ([We07]). Let $\mathcal{M}=(M, \leq,+, \ldots)$ be a weakly o-minimal structure expanding an ordered group $(M, \leq,+)$. Then $\mathcal{M}$ is of non-valuational type iff $\mathcal{M}$ has the strong cell decomposition property.

2. The main result. Throughout this section we shall work in a weakly o-minimal structure $\mathcal{M}=(M, \leq, \ldots)$, usually assuming that $\mathcal{M}$ has the strong cell decomposition property or is a non-valuational expansion of an ordered group. Let $C$ denote a non-empty, convex and non-definable (in $\mathcal{M}$ ) proper subset of $M$ such that $\inf C=-\infty$. Then $\langle C, M \backslash C\rangle$ is a cut in $(M, \leq)$ (in $[\mathrm{Bz}]$ and $[\mathrm{BVT}]$ such cuts are called irrational). By Corollary 2.15 from [We07], we know that if $\mathcal{M}$ is a non-valuational expansion of an ordered group, then $\operatorname{Th}(\mathcal{M})$ is weakly o-minimal. Hence, by [Bz], also $\operatorname{Th}(\mathcal{M}, C)$ is weakly o-minimal. In a series of lemmas below we will show that if $\mathcal{M}$ is a weakly o-minimal non-valuational expansion of an ordered group and the cut $\langle C, M \backslash C\rangle$ is non-valuational, then also the expansion $(\mathcal{M}, C)$ is of non-valuational type. Moreover, we will give a direct proof of the weak o-minimality of $\operatorname{Th}(\mathcal{M}, C)$. 
For $m \in \mathbb{N}_{+}$and $i \in\{1, \ldots, m+1\}$, we denote by $\pi_{i}^{m+1}$ the projection from $M^{m+1}$ to $M^{m}$ dropping the $i$ th coordinate, and by $\mathcal{D}_{m}(\mathcal{M})$ the family of all subsets of $M^{m}$ definable in $\mathcal{M}$.

Lemma 2.1. Assume that $\mathcal{M}=(M, \leq, \ldots)$ is a weakly o-minimal structure and $I \subseteq M$ is a convex open definable set such that $I \cap C \neq \emptyset$ and $I \backslash C \neq \emptyset$. If $f: I \rightarrow \bar{M}^{\mathcal{M}}$ is a definable function such that $(\forall x \in I)(f(x)>c)$ $[(\forall x \in I)(f(x)<c)]$ for some $c \in M$, then there are an open interval $J \subseteq I$ and $\alpha>c[\alpha<c], \alpha \in M$, such that $J \cap C \neq \emptyset, J \backslash C \neq \emptyset$ and $(\forall x \in J)(f(x)>\alpha)[(\forall x \in J)(f(x)<\alpha)]$.

Proof. Let $f: I \rightarrow \bar{M}^{\mathcal{M}}$ be a definable function such that $(\forall x \in I)$ $(f(x)>c)$, where $c \in M$. By Fact 1.2 and the non-definability of $C$, there is an open interval $I_{1} \subseteq I$ such that $I_{1} \cap C \neq \emptyset, I_{1} \backslash C \neq \emptyset$ and $f\left\lceil I_{1}\right.$ is either strictly monotone or constant. Fix $a \in I_{1} \cap C, b \in I_{1} \backslash C$ and $\alpha \in M$ such that $c<\alpha<\min \{f(a), f(b)\}$. It is clear that $f(x)>\alpha$ whenever $x \in(a, b)$. The other case is proved in a similar way.

Lemma 2.2. Let $\mathcal{M}=(M, \leq, \ldots)$ be a weakly o-minimal non-valuational expansion of an ordered group. Assume that $I \subseteq M$ is a non-empty convex open definable set such that $I \cap C \neq \emptyset$ and $I \backslash C \neq \emptyset$, and $f, g: I \rightarrow \bar{M}^{\mathcal{M}}$ are definable functions such that $(\forall x \in I)(f(x)<g(x))$. Then there are an element $a \in M$ and an open interval $J \subseteq I$ such that $J \cap C \neq \emptyset, J \backslash C \neq \emptyset$ and $f(x)<a<g(x)$ for $x \in J$.

Proof. By Theorem 1.4 and Fact 1.3, without loss of generality we can assume that the functions $f, g$ are strongly continuous. By Lemma 2.1, there are an open interval $I_{1} \subseteq I$ and an element $\alpha>0$ in $M$ such that $I_{1} \cap C \neq \emptyset$, $I_{1} \backslash C \neq \emptyset$ and $\left(\forall x \in I_{1}\right)(g(x)-f(x)>\alpha)$. Fix $\varepsilon>0$ in $M$ such that $2 \varepsilon<\alpha$. For $x_{0} \in I_{1}$ define

$$
\begin{aligned}
\delta_{f}\left(x_{0}\right)=\min (\varepsilon, \sup \{d \in & M: d>0, \\
& \left.\left.\left(\forall x \in\left(x_{0}-d, x_{0}+d\right) \cap I_{1}\right)\left(\left|f(x)-f\left(x_{0}\right)\right|<\varepsilon\right)\right\}\right), \\
\delta_{g}\left(x_{0}\right)=\min (\varepsilon, \sup \{d \in M: d>0, & \left.\left.\left(\forall x \in\left(x_{0}-d, x_{0}+d\right) \cap I_{1}\right)\left(\left|g(x)-g\left(x_{0}\right)\right|<\varepsilon\right)\right\}\right) .
\end{aligned}
$$

Again, by Lemma 2.1, there are an open interval $I_{2} \subseteq I_{1}$ and an element $\beta>0$ in $M$ such that $I_{2} \cap C \neq \emptyset, I_{2} \backslash C \neq \emptyset$ and $\min \left(\delta_{f}\left(x_{0}\right), \delta_{g}\left(x_{0}\right)\right)>\beta$ for $x_{0} \in I_{2}$. Fix $c_{1} \in I_{2} \cap C$ and $c_{2} \in I_{2} \backslash C$ such that $c_{2}-c_{1}<\beta$. For $x_{1}, x_{2} \in\left(c_{1}, c_{2}\right)$ we have

$$
g\left(x_{2}\right)-f\left(x_{1}\right)=\left(g\left(x_{2}\right)-g\left(x_{1}\right)\right)+\left(g\left(x_{1}\right)-f\left(x_{1}\right)\right)>-\varepsilon+2 \varepsilon=\varepsilon .
$$

Consequently,

$$
\inf \left\{g(x): x \in\left(c_{1}, c_{2}\right)\right\}-\sup \left\{f(x): x \in\left(c_{1}, c_{2}\right)\right\} \geq \varepsilon .
$$


If $a \in M$ is such that $\sup \left\{f(x): x \in\left(c_{1}, c_{2}\right)\right\}<a<\inf \left\{g(x): x \in\left(c_{1}, c_{2}\right)\right\}$, then for $x \in\left(c_{1}, c_{2}\right)$ we have $f(x)<a<g(x)$.

LEMma 2.3. Let $\mathcal{M}$ be a weakly o-minimal non-valuational expansion of an ordered group. Assume that $X \subseteq M^{2}$ is a set definable in $\mathcal{M}$ such that for any $a, b \in M$, if $\langle a, b\rangle \in X$, then there are $a_{1}, a_{2} \in M$ such that $a_{1}<a<a_{2}$ and $\left(a_{1}, a_{2}\right) \times\{b\} \subseteq X$. The following conditions are equivalent.

(a) There are $a_{1} \in C, a_{2} \in M \backslash C$ and $b \in M$ such that $\left(a_{1}, a_{2}\right) \times\{b\} \subseteq X$.

(b) There are $a_{1} \in C$ and $a_{2} \in M \backslash C$ such that $\left(a_{1}, a_{2}\right) \subseteq \pi_{2}^{2}[X]$.

Proof. The implication (a) $\Rightarrow$ (b) is obvious, so assume that (b) holds. Let $\mathcal{C}$ be a decomposition of $M^{2}$ into strong cells in $M^{2}$ partitioning the set $X$. There is a convex open definable set $I \subseteq M$ such that $I \cap C \neq \emptyset, I \backslash C \neq \emptyset$ and for every $D \in \mathcal{C}$, either $I=\pi_{2}^{2}[D]$ or $\pi_{2}^{2}[D] \cap I=\emptyset$. Below we consider two cases.

CASE 1: The set $(I \times M) \cap X$ has empty interior. The following claim is a consequence of Lemma 2.1 from [We06] but for the sake of completeness we give the proof in our particular situation.

Claim. For every $x \in I$, the set $\{y \in M:\langle x, y\rangle \in X\}$ is finite.

Proof of the Claim. Suppose for a contradiction that for some $a \in I$, the set $\{y \in M:\langle a, y\rangle \in X\}$ is infinite, so it contains an open interval $J$. For $b \in J$ define

$$
f(b)=\sup \{c \in M: c>a \text { and }\{a\} \times(b, c) \subseteq(I \times M) \cap X\} .
$$

The function $f$ assumes values greater than $a$ in $\bar{M}^{\mathcal{M}} \cup\{+\infty\}$. By Fact 1.3 , there is an open interval $J^{\prime} \subseteq J$ such that $f \nmid J^{\prime}$ is strongly continuous and strictly monotone or constant. It is clear that the set $\{\langle x, y\rangle: a<x<$ $\left.f(y), y \in J^{\prime}\right\}$ is contained in $(I \times M) \cap X$ and contains an open box itself. This means that $(I \times M) \cap X$ has non-empty interior, a contradiction.

Using the Claim, for $x \in I$, we can define $f(x)=\min \{y \in M:\langle x, y\rangle \in X\}$. Our assumptions guarantee that $f$ is constant (say $f(x)=b$ whenever $x \in I$ ), so for any $a_{1} \in I \cap C$ and $a_{2} \in I \backslash C$, we have $\left(a_{1}, a_{2}\right) \times\{b\} \subseteq X$.

CASE 2: There are definable strongly continuous functions $f: I \rightarrow$ $\bar{M}^{\mathcal{M}} \cup\{-\infty\}$ and $g: I \rightarrow \bar{M}^{\mathcal{M}} \cup\{+\infty\}$ such that $\bar{f}(a)<\bar{g}(a)$ for $a \in \bar{I}$ and $D:=(f, g)_{I} \subseteq X \cap(I \times M)$. By Lemma 2.2, there are $a_{1} \in I \cap C, a_{2} \in I \backslash C$ and $b \in M$ such that $\left(a_{1}, a_{2}\right) \times\{b\} \subseteq D \subseteq X$.

For a weakly o-minimal structure $\mathcal{M}$ and a set $X \in \mathcal{D}_{m+1}(\mathcal{M})$ let

$$
I(X, C)=\left\{\bar{a} \in M^{m}:(\exists b \in C)(\exists c \in M \backslash C)(\{\bar{a}\} \times(b, c) \subseteq X)\right\} .
$$

Define also $\mathcal{E}_{m}(\mathcal{M}, C)=\left\{I(X, C): X \in \mathcal{D}_{m+1}(\mathcal{M})\right\}$. Below we will show that if $\mathcal{M}$ is a non-valuational expansion of an ordered group, then $\mathcal{E}_{m}(\mathcal{M}, C)$ is exactly the family of subsets of $M^{m}$ definable in $(\mathcal{M}, C)$. 
Lemma 2.4. Let $\mathcal{M}$ be a weakly o-minimal structure with the strong cell decomposition property.

(a) If $X \in \mathcal{D}_{2}(\mathcal{M})$ is a strong cell, then $I\left(\left\{\langle x, y\rangle \in M^{2}:\langle y, x\rangle \in X\right\}, C\right)$ is a convex set.

(b) If $Y \in \mathcal{E}_{1}(\mathcal{M}, C)$, then $Y$ is a finite union of convex sets.

(c) $C \in \mathcal{E}_{1}(\mathcal{M}, C)$.

Proof. (a) is obvious from the definition of strong cells. (b) follows from (a) and the strong cell decomposition property of $\mathcal{M}$. For the proof of (c), note that

$$
C=I\left(\left\{\langle x, y\rangle \in M^{2}: y>x\right\}, C\right) .
$$

Here the strong cell decomposition property in not needed.

LEMma 2.5. Assume that $\mathcal{M}$ is a weakly o-minimal structure and $m \in \mathbb{N}_{+}$.

(a) $\mathcal{D}_{m}(\mathcal{M}) \subseteq \mathcal{E}_{m}(\mathcal{M}, C)$.

(b) $\mathcal{E}_{m}(\mathcal{M}, C)$ is closed under Boolean operations.

(c) If $X \in \mathcal{E}_{m}(\mathcal{M}, C)$, then $X \times M, M \times X \in \mathcal{E}_{m+1}(\mathcal{M}, C)$.

Proof. (a) If $m \in \mathbb{N}_{+}$and $X \in \mathcal{D}_{m}(\mathcal{M})$, then $X=I(X \times M, C)$.

(b) Fix $m \in \mathbb{N}_{+}$and $X, Y \in \mathcal{E}_{m}(\mathcal{M}, C)$. There are $X_{1}, Y_{1} \in \mathcal{D}_{m+1}(\mathcal{M})$ such that $X=I\left(X_{1}, C\right)$ and $Y=I\left(Y_{1}, C\right)$. Clearly, $X \cup Y=I\left(X_{1} \cup Y_{1}, C\right)$, $X \cap Y=I\left(X_{1} \cap Y_{1}, C\right)$ and $M^{m} \backslash X=I\left(M^{m+1} \backslash X_{1}, C\right)$.

(c) Let $m \in \mathbb{N}_{+}$and $X \in \mathcal{E}_{m}(\mathcal{M}, C)$. Then $X=I\left(X_{1}, C\right)$ for some $X_{1} \in \mathcal{D}_{m+1}(\mathcal{M})$. Hence $M \times X=I\left(M \times X_{1}, C\right)$ and $X \times M=I(\{\langle\bar{x}, y, z\rangle \in$ $\left.\left.M^{m+1}:\langle\bar{x}, z\rangle \in X\right\}, C\right)$.

LEMma 2.6. Let $\mathcal{M}$ be a weakly o-minimal expansion of an ordered group. If $X \in \mathcal{E}_{m+1}(\mathcal{M}, C)$, then $\pi_{m+1}^{m+1}[X] \in \mathcal{E}_{m}(\mathcal{M}, C)$.

Proof. Fix $m \in \mathbb{N}_{+}$and $X \in \mathcal{E}_{m+1}(\mathcal{M}, C)$. There is $X_{1} \in \mathcal{D}_{m+2}(\mathcal{M})$ such that $X=I\left(X_{1}, C\right)$. Let

$$
X_{2}=\bigcup\left\{\{\bar{a}\} \times(b, c): \bar{a} \in M^{m+1}, b \in C, c \in M \backslash C \text { and }\{\bar{a}\} \times(b, c) \subseteq X_{1}\right\} .
$$

Clearly, $X=I\left(X_{2}, C\right)$ and $X_{2} \in \mathcal{D}_{m+2}(\mathcal{M})$. We claim that $\pi_{m+1}^{m+1}[X]=$ $I\left(\pi_{m+1}^{m+2}\left[X_{2}\right], C\right)$.

In order to prove that $\pi_{m+1}^{m+1}[X] \subseteq I\left(\pi_{m+1}^{m+2}\left[X_{2}\right], C\right)$, fix $\bar{a} \in \pi_{m+1}^{m+1}[X]$. There is $b \in M$ such that $\langle\bar{a}, b\rangle \in X=I\left(X_{2}, C\right)$. So there are $c \in C$ and $d \in M \backslash C$ such that $\{\langle\bar{a}, b\rangle\} \times(c, d) \subseteq X_{2}$. Hence $\{\bar{a}\} \times(c, d) \subseteq \pi_{m+1}^{m+2}\left[X_{2}\right]$ and $\bar{a} \in I\left(\pi_{m+1}^{m+2}\left[X_{2}\right], C\right)$.

For the reverse inclusion, let $\bar{a} \in I\left(\pi_{m+1}^{m+2}\left[X_{2}\right], C\right)$. There are $c \in C$ and $d \in M \backslash C$ such that $\{\bar{a}\} \times(c, d) \subseteq \pi_{m+1}^{m+2}\left[X_{2}\right]$. Let $Z=\left\{\langle x, y\rangle \in M^{2}\right.$ : $\left.\langle\bar{a}, y, x\rangle \in X_{2}\right\}$. Clearly, $(c, d) \subseteq \pi_{2}^{2}[Z]$. By Lemma 2.3, there are $c^{\prime} \in C$, 
$d^{\prime} \in M \backslash C$ and $e \in M$ such that $(c, d) \times\{e\} \subseteq Z$. Consequently, $\{\langle\bar{a}, e\rangle\} \times$ $(c, d) \subseteq X_{2}$. The latter implies that $\langle\bar{a}, e\rangle \in X$ and $\bar{a} \in \pi_{m+1}^{m+1}[X]$.

Lemmas $2.4-2.6$ imply that if $\mathcal{M}$ is a weakly o-minimal non-valuational expansion of an ordered group, then:

(a) all intervals in $(M, \leq)$ belong to $\mathcal{E}_{1}(\mathcal{M}, C)$;

(b) every set belonging to $\mathcal{E}_{1}(\mathcal{M}, C)$ is a union of finitely many convex sets;

(c) $\left\{\langle x, y\rangle \in M^{2}: x<y\right\} \in \mathcal{E}_{2}(\mathcal{M}, C)$;

(d) for every $m \in \mathbb{N}_{+}, \emptyset, M^{m} \in \mathcal{E}_{m}(\mathcal{M}, C)$ and $\left(\mathcal{E}(\mathcal{M}, C), \cap, \cup,^{c}\right)$ is a Boolean algebra;

(e) if $X \in \mathcal{E}_{m}(\mathcal{M}, C)$, then $X \times M, M \times X \in \mathcal{E}_{m+1}(\mathcal{M}, C)$;

(f) if $1 \leq i<j \leq m$, then $\left\{\left\langle x_{1}, \ldots, x_{m}\right\rangle \in M^{m}: x_{i}=x_{j}\right\} \in \mathcal{E}_{m}(\mathcal{M}, C)$;

(g) if $X \in \mathcal{E}_{m+1}(\mathcal{M}, C)$ and $i \in\{1, \ldots, m\}$, then $\pi_{i}^{m+1}[X] \in \mathcal{E}_{m}(\mathcal{M}, C)$;

(h) $C \in \mathcal{E}_{1}(\mathcal{M}, C)$.

Therefore, by Remark 1.1, there is a weakly o-minimal structure $\mathcal{M}^{\prime}$ expanding $\mathcal{M}$ such that a set $X \subseteq M^{m}$ is definable in $\mathcal{M}^{\prime}$ iff $X \in \mathcal{E}_{m}(\mathcal{M}, C)$. In the following lemma, $\mathcal{D}_{m}(\mathcal{M}, C)$ denotes the family of all subsets of $M^{m}$ which are definable in the structure $(\mathcal{M}, C)$.

Lemma 2.7. Let $\mathcal{M}$ be a weakly o-minimal non-valuational expansion of an ordered group. For every $m \in \mathbb{N}_{+}, \mathcal{E}_{m}(\mathcal{M}, \mathcal{C})=\mathcal{D}_{m}(\mathcal{M}, C)$.

Proof. The inclusion $\subseteq$ is obvious. That $\mathcal{D}_{m}(\mathcal{M}, C) \subseteq \mathcal{E}_{m}(\mathcal{M}, C)$ follows easily by induction from the above remark.

COROLlary 2.8. If $\mathcal{M}$ is a weakly o-minimal non-valuational expansion of an ordered group, then $(\mathcal{M}, C)$ is weakly o-minimal.

LEMma 2.9. Let $\mathcal{M}=(M, \leq,+, \ldots)$ be a weakly o-minimal non-valuational expansion of an ordered group $(M, \leq,+)$. Assume that $I$ is a non-empty convex open definable $($ in $\mathcal{M})$ set with $I \cap C \neq \emptyset$ and $I \backslash C \neq \emptyset$. Let $f: I \rightarrow$ $\bar{M}^{\mathcal{M}}$ be a definable strongly continuous and strictly monotone function.

(a) If $f$ is strictly increasing, then

$$
\langle\{a \in M:(\exists c \in C)(f(c)>a)\},\{a \in M:(\exists d \in M \backslash C)(f(d)<a)\}\rangle
$$

is a non-definable and non-valuational cut in $(M, \leq,+)$.

(b) If $f$ is strictly decreasing, then

$$
\langle\{a \in M:(\exists d \in M \backslash C)(f(d)>a)\},\{a \in M:(\exists c \in C)(f(c)<a)\}\rangle
$$

is a non-definable and non-valuational cut in $(M, \leq,+)$.

Proof. As both cases are similar, we will only prove (a). Assume that $f$ is strictly increasing and let

$$
C^{\prime}=\{a \in M:(\exists c \in C)(f(c)>a)\}
$$


and

$$
D^{\prime}=\{a \in M:(\exists d \in M \backslash C)(f(d)<a)\} .
$$

It is clear that $C^{\prime}$ and $D^{\prime}$ are both convex definable sets with inf $C^{\prime}=-\infty$ and $\sup D^{\prime}=+\infty$. The sets $C^{\prime}, D^{\prime}$ are disjoint since otherwise we would have $f(d)<a<f(c)$ for some $a \in M, c \in C$ and $d \in M \backslash C$.

To show that $C^{\prime} \cup D^{\prime}=M$, suppose for a contradiction that there exists an element $a \in M \backslash\left(C^{\prime} \cup D^{\prime}\right)$. This means that $f(c) \leq a \leq f(d)$ whenever $c \in C$ and $d \in M \backslash C$. Note that if there was a $c \in C$ with $f(c)=a$, then there would also be a $c^{\prime} \in C$ with $c^{\prime}>c$ and $f\left(c^{\prime}\right)>f(c)=a$, a contradiction. So $(\forall c \in C)(f(c)<a)$ and similarly $(\forall d \in M \backslash C)(f(d)>a)$. Now,

$$
C=\{x \in M: x \leq \inf I\} \cup\{x \in I: f(x)<a\},
$$

which means that $C$ is definable in $\mathcal{M}$, a contradiction. In this way we have shown that $\left\langle C^{\prime}, D^{\prime}\right\rangle$ is a cut in $(M, \leq)$. Its non-definability is a consequence of the non-definability of $C$.

In order to complete the proof, suppose for a contradiction that

$$
\inf \left\{z-y: y \in C^{\prime}, z \in D^{\prime}\right\}=\inf \{f(d)-f(c): c \in C, d \in M \backslash C\}>0
$$

and fix $\varepsilon>0$ in $M$ such that $\varepsilon<\inf \{f(d)-f(c): c \in C, d \in M \backslash C\}$. So clearly $f\left(x_{2}\right)-f\left(x_{1}\right)>\varepsilon$ whenever $x_{1} \in I \cap C$ and $x_{2} \in I \backslash C$. For $x_{0} \in I$ define

$$
\begin{aligned}
\delta\left(x_{0}\right)=\min \{\varepsilon, \sup \{d \in M: d>0 \text { and } & \left|f(x)-f\left(x_{0}\right)\right|<\varepsilon \\
& \text { for } \left.\left.x \in\left(x_{0}-d, x_{0}+d\right) \cap I\right\}\right\} .
\end{aligned}
$$

By Lemma 2.1, there are an open interval $J \subseteq I$ and an element $\alpha>0$ in $M$ such that $J \cap C \neq \emptyset, J \backslash C \neq \emptyset$ and $\left(\forall x_{0} \in J\right)\left(\delta\left(x_{0}\right)>\alpha\right)$. Since the cut $\langle C, M \backslash C\rangle$ is non-valuational, we can choose $x_{1} \in J \cap C$ and $x_{2} \in J \backslash C$ so that $x_{2}-x_{1}<\alpha$. We then have $\delta\left(x_{1}\right)>\alpha$ and $\left|f\left(x_{2}\right)-f\left(x_{1}\right)\right|<\varepsilon$, which contradicts our choice of $\varepsilon$.

Corollary 2.10. Let $\mathcal{M}$ be a weakly o-minimal non-valuational expansion of an ordered group.

(a) The structure $(\mathcal{M}, C)$ is of non-valuational type.

(b) $\operatorname{Th}(\mathcal{M}, C)$ is weakly o-minimal.

Proof. We already know by Corollary 2.8 that $(\mathcal{M}, C)$ is weakly o-minimal. To demonstrate that $(\mathcal{M}, C)$ is of non-valuational type, fix a cut $\left\langle D, D^{\prime}\right\rangle$ in $(M, \leq)$ which is definable in $(\mathcal{M}, C)$. We have to show that $\left\langle D, D^{\prime}\right\rangle$ is nonvaluational. As there is nothing to do in case $D \in \mathcal{D}_{1}(\mathcal{M})$, suppose that $D$ is not definable in $\mathcal{M}$. By Lemma 2.7, there exists a set $X \in \mathcal{D}_{2}(\mathcal{M})$ such that $D=I(X, C)$. Denote by $X^{\prime}$ the union of all sets of the form $\{a\} \times(b, c)$, where $a, b, c \in M, b<c$ and $\{a\} \times(b, c) \subseteq X$. Obviously, $X^{\prime}$ is definable in $\mathcal{M}$ and $I\left(X^{\prime}, C\right)=D$. Fix a decomposition $\mathcal{C}$ of $M^{2}$ into strong cells 
partitioning $X^{\prime}$ and let $I_{0}, \ldots, I_{n}$ be an enumeration of all convex sets of the form $\pi_{2}^{2}[Y]$, where $Y \in \mathcal{C}$ and $Y \subseteq X^{\prime}$, such that $I_{i}$ precedes $I_{j}$ whenever $i<j \leq n$. Without loss of generality we can assume that the functions appearing in definitions of cells from $\mathcal{C}$ are strictly monotone or constant. Non-definability of $D$ guarantees that there is $k \leq n$ such that $I_{k}$ is a convex open set intersecting both $D$ and $M \backslash D$. There are strongly continuous functions $f_{0}, g_{0}, \ldots, f_{m}, g_{m}: I_{k} \rightarrow \bar{M}^{\mathcal{M}} \cup\{-\infty,+\infty\}$ such that

(a) each of $f_{0}, g_{0}, \ldots, f_{m}, g_{m}$ is either strictly monotone or constant;

(b) $\bar{f}_{i}(x)<\bar{g}_{i}(x)$ for $i \leq m$ and $x \in \bar{I}_{k}$;

(c) $\bar{g}_{i}(x)<\bar{f}_{i+1}(x)$ for $i<m$ and $x \in \bar{I}_{k}$;

(d) $X^{\prime} \cap\left(I_{k} \times M\right)=\left(f_{0}, g_{0}\right)_{I_{k}} \cup \cdots \cup\left(f_{m}, g_{m}\right)_{I_{k}}$.

There is a unique $i \leq m$ such that $\sup I\left(\left(f_{i}, g_{i}\right)_{I_{k}}, C\right)=\sup D \cap I_{k}$ and exactly one of the following two conditions holds.

(1) $f_{i}$ is strictly increasing and $D \cap I_{k}=I\left(\left(f_{i},+\infty\right)_{I_{k}}, C\right)$.

(2) $g_{i}$ is strictly decreasing and $D \cap I_{k}=I\left(\left(-\infty, g_{i}\right)_{I_{k}}, C\right)$.

Since the reasoning is similar in both cases, we will only consider (1). To simplify notation let $f:=f_{i}$. Fix $a \in C$ such that $a>\inf \left\{f(x): x \in I_{k}\right\}$ and $b \in M \backslash C$ such that $b<\sup \left\{f(x): x \in I_{k}\right\}$, and let $J=(a, b)$. For $y \in J$ define

$$
h(y)=\sup \left\{x \in M:\langle x, y\rangle \in(f,+\infty)_{I_{k}}\right\} .
$$

The function $h$ is strictly increasing and strongly continuous. As

$$
D=\left\{x \in M:\left(\exists x^{\prime} \in C\right)\left(h\left(x^{\prime}\right)>x\right)\right\},
$$

by Lemma 2.9 the cut $\left\langle D, D^{\prime}\right\rangle$ is non-valuational.

The weak o-minimality of $\operatorname{Th}(\mathcal{M}, C)$ now follows from the fact that $(\mathcal{M}, C)$ is a weakly o-minimal non-valuational structure and from Corollary 2.15 from [We07].

Before formulating the main result, we will introduce so called unary nonvaluational predicates. Assume that $\mathcal{M}=(M, \leq, \ldots)$ is a weakly o-minimal non-valuational expansion of an ordered group and let $X \subseteq M$ be a finite union of convex sets. For $a \in X$ denote by $R(a, X)$ the convex component of $X$ containing $a$. Similarly, for $a \in M \backslash X$, let $R(a, X)$ be the convex component of $M \backslash X$ containing $a$. For $a \in M$ define

$$
D(a, X)=\bigcup_{\alpha \in R(a, X)}(\alpha,+\infty) .
$$

Clearly, if $D(a, X) \neq M$, then $\langle M \backslash D(a, X), D(a, X)\rangle$ is a cut in $(M, \leq)$. A cut $\langle C, D\rangle$ in $(M, \leq)$ is said to be determined by $X$ if it is of the form $\langle M \backslash D(a, X), D(a, X)\rangle$ for some $a \in M$. 
We say that a set $X \subseteq M$ is a unary non-valuational predicate iff $X$ is a union of finitely many convex sets and all cuts determined by $X$ are non-valuational.

THeOREM 2.11. Assume that $\mathcal{M}$ is a weakly o-minimal non-valuational expansion of an ordered group and $\mathcal{N}$ is an expansion of $\mathcal{M}$ by a family of non-valuational unary predicates. Then $\mathcal{N}$ is of non-valuational type.

Proof. There is a family of convex open sets $C_{i} \subsetneq M, i \in I$, such that

- for every $i \in I, C_{i}$ is not definable in $\mathcal{M}$ and $\inf C_{i}=-\infty$;

- the structures $\mathcal{N}$ and $\left(\mathcal{M}, C_{i}: i \in I\right)$ have the same definable sets.

Without loss of generality we can assume that $I$ is finite, in which case the theorem follows easily by induction on $|I|$ from Corollary 2.10(b).

Theorem 2.11 actually shows that for weakly o-minimal expansions of ordered groups, the property of a structure having the strong cell decomposition is preserved under expansions by families of unary non-valuational predicates. Having in mind that non-valuational predicates are those which determine non-valuational cuts, one can speak of valuational/non-valuational cuts in an arbitrary weakly o-minimal structure with the strong cell decomposition property, not necessarily expanding an ordered group. More precisely, if $\mathcal{M}=(M, \leq, \ldots)$ is a weakly o-minimal structure with the strong cell decomposition property, then a cut $\langle C, D\rangle$ in $(M, \leq)$ could be called non-valuational if the structure $(\mathcal{M}, C)$ has the strong cell decomposition property. This gives us notions of being "close" and being "far" for the parts of a cut $\langle C, D\rangle$ in $(M, \leq)$ and it would be interesting to further investigate this topic, probably relating it to the canonical o-minimal extension of a weakly o-minimal structure with the strong cell decomposition property constructed in [We07].

\section{References}

[Ar] R. D. Aref'ev, On the monotonicity property for weakly o-minimal models, in: Algebra and Model Theory, Novosibirsk. Gos. Tekh. Univ., Novosibirsk, 1997, 8-15 (in Russian); Model Theory in Kazakhstan, Eco Stydy, Almaty, 2006, 7-13 (in Russian).

[BP] Y. Baisalov et B. Poizat, Paires de structures o-minimales, J. Symbolic Logic 63 (1998), 570-578.

[Bz] B. S. Baizhanov, Expansion of a model of a weakly o-minimal theory by a family of unary predicates, ibid. 66 (2001), 1382-1414.

[BVT] B. S. Baizhanov, V. Verbovskiǐ and G. Turekhanova, Non-orthogonality of 1types in weakly o-minimal structures of finite depth, preprint (in Russian).

[D1] L. van den Dries, Tame Topology and o-Minimal Structures, London Math. Soc. Lecture Note Ser. 248, Cambridge Univ. Press, Cambridge,1998.

[D2] - , T-convexity and tame extensions, II, J. Symbolic Logic 62 (1997), 14-34. 
[DL] L. van den Dries and A. H. Lewenberg, T-convexity and tame extensions, ibid. 60 (1995), 74-102.

[MMS] D. Macpherson, D. Marker, and C. Steinhorn, Weakly o-minimal structures and real closed fields, Trans. Amer. Math. Soc. 352 (2000), 5435-5483.

[Sh783] S. Shelah, Dependent first order theories, continued, Israel J. Math., to appear.

[We06] R. Wencel, Topological properties of sets definable in weakly o-minimal structures, J. Symbolic Logic, submitted.

[We07] —, Weakly o-minimal non-valuational structures, Ann. Pure Appl. Logic 154 (2008), 139-162.

Mathematical Institute

University of Wrocław

pl. Grunwaldzki 2/4

50-384 Wrocław, Poland

E-mail: rwenc@math.uni.wroc.pl

Received 29 January 2008;

in revised form 11 July 2008 